\title{
ON THE EXISTENCE OF FIXPOINTS OF COMPOSITE MEROMORPHIC FUNCTIONS
}

\author{
WALTER BERGWEILER \\ (Communicated by Clifford J. Earle, Jr.)
}

\begin{abstract}
Let $f$ be a meromorphic function with at least two poles, and let $g$ be an entire transcendental function. It is proved that the composite function $f \circ g$ has infinitely many fixpoints.
\end{abstract}

\section{INTRODUCTION AND MAIN RESULT}

Let $f$ be a transcendental meromorphic function, and let $g$ be a transcendental entire function. Gross [3, p. 247, Problem 6] has asked whether the composite function $f \circ g$ has infinitely many fixpoints. This was proved in [1] if $f$ is entire, and it can be proved by the same method if $f$ has exactly one pole. In this note, we shall give an affirmative answer to Gross's question.

Theorem. Let $f$ be a meromorphic function that has at least two different poles, and let $g$ be a transcendental entire function. Then the composite function $f \circ g$ has infinitely many fixpoints.

The proof is very short and based on Picard's theorem, but completely elementary otherwise. In fact, the argument is quite simple, but it seems to have been overlooked before.

\section{LEMMAS}

We shall use the following elementary lemmas.

Lemma 1. Let $f$ be a meromorphic function, and let $z_{0}$ be a pole of $f$ of order $p$. Then there exists a function $h$, defined and analytic in a neighborhood of 0 , such that $h(0)=0$ and $f\left(h(z)+z_{0}\right)=z^{-p}$ for $z \neq 0$.

To prove Lemma 1 , we note that there exists a function $k$, defined and analytic in a neighborhood of 0 , such that $f\left(z+z_{0}\right)=k(z)^{-p}$ and $k^{\prime}(0) \neq 0$. The conclusion follows if we define $h$ as the inverse function of $k$.

Received by the editors May 8, 1990 .

1991 Mathematics Subject Classification. Primary 30D05.

Key words and phrases. Entire function, meromorphic function, composite function, fixpoint.

Presented to the Deutsche Mathematiker-Vereinigung Bremen, September 16-22, 1990. 
Lemma 2. Let $f$ and $g$ be meromorphic functions. Then $f \circ g$ has infinitely many fixpoints if and only if $g \circ f$ does.

Lemma 2 follows from the fact that $g$ maps the set of fixpoints of $f \circ g$ bijectively onto the set of fixpoints of $g \circ f$. Lemma 2 is not new and has been used in several papers on the subject. It seems to have been noticed first by Gross [2, Lemma 3].

\section{Proof of the Theorem}

Let $z_{1}$ and $z_{2}$ be poles of $f$. For $j \in\{1,2\}$, denote the order of $z_{j}$ by $p_{j}$ and choose $h_{j}$ according to Lemma 1 . Define $k_{1}(z)=h_{1}\left(z^{p_{2}}\right)+z_{1}$ and $k_{2}(z)=$ $h_{2}\left(z^{p_{1}}\right)+z_{2}$. Then $f\left(k_{1}(z)\right)=f\left(k_{2}(z)\right)=z^{-p_{1} p_{2}}$ in a punctured neighborhood of 0 . Now define $u(z)=g\left(z^{-p_{1} p_{2}}\right)$. Then 0 is an essential singularity of $u$ and in a punctured neighborhood of 0 we have $u(z)=g\left(f\left(k_{1}(z)\right)\right)=g\left(f\left(k_{2}(z)\right)\right)$.

Suppose now that $f \circ g$ has only finitely many fixpoints. Then $g \circ f$ has only finitely many fixpoints by Lemma 2 . It follows that $u(z) \neq k_{j}(z)$ for $j \in\{1,2\}$ in a punctured neighborhood of 0 . Moreover, $k_{1}(z) \neq k_{2}(z)$ in a neighborhood of 0 since $k_{1}(0)=z_{1} \neq z_{2}=k_{2}(0)$. Define

$$
v(z)=\frac{u(z)-k_{1}(z)}{k_{2}(z)-k_{1}(z)} \text {. }
$$

Then 0 is an essential singularity of $v$ and $v$ does not take the values 0,1 , and $\infty$ in a punctured neighborhood of 0 . This contradicts Picard's Theorem and the theorem is proved.

\section{REMARK}

Similarly, we can prove that if $f$ and $g$ are transcendental meromorphic functions and if one of the two functions $f$ and $g$ has at least three poles, then $f \circ g$ has infinitely many fixpoints.

\section{REFERENCES}

1. W. Bergweiler, Proof of a conjecture of Gross concerning fix-points, Math. Z. 204 (1990), 381-390.

2. F. Gross, On factorization of meromorphic functions, Trans. Amer. Math. Soc. 131 (1968), 215-222.

3. Factorization of meromorphic functions, U.S. Government Printing Office, Washington, DC, 1972.

Lehrstuhl II für Mathematik, RWTH Aachen, Templergraben 55, D-5100 Aachen, Federal Republic of Germany

E-mail address: sf010be@dacth11.bitnet 